\title{
Communicable Diseases Report, NSW, January and February 2008
}

\section{Communicable Diseases Branch, NSW Department of Health}

For updated information, including data and facts on specific diseases, visit www.health.nsw.gov.au and click on Infectious Diseases.

The communicable diseases site http://www.health. nsw.gov.au/publichealth/infectious/index.asp has been reborn using browser-friendly html formats to improve accessibility and as a result has a new address.

Figure 1 and Tables 1 and 2 show reports of communicable diseases received through to the end of February 2008 in New South Wales (NSW).

\section{Measles outbreak}

One case

In early January 2008, South Eastern Sydney Illawarra Public Health Unit (PHU) reported a confirmed measles case in a 12-year-old boy. The boy developed a fever of $38.5^{\circ} \mathrm{C}$, sore throat and a slight cough. Two days later a rash appeared on his forehead, spreading to his face and behind his ears, and his temperature was reported as $38^{\circ} \mathrm{C}$. The fever eased and the rash progressed to his trunk. Although the family reported no specific contact with other cases of measles, while overseas he did have contact with another child with a few spots on his hand thought to be caused by a viral illness. The child had returned from overseas by aeroplane during his infectious period (from just before onset of symptoms to 4 days after onset of the rash). The child had reportedly had two measles vaccinations some years before.

Measles is an acute viral disease that can have serious complications. In the past, measles infection was common in childhood. As a result of immunisation, measles is now rare in NSW. ${ }^{1}$ The first symptoms of infection with measles include fever, tiredness, runny nose, cough and sore red eyes. These symptoms usually last for a few days before a red blotchy rash appears. The rash starts on the face and upper neck over 1 to 2 days, spreads down the body and usually lasts for 4 to 7 days. ${ }^{2}$
Measles is a notifiable disease in NSW and must be reported as soon as possible after diagnosis. PHUs investigate cases to identify possible epidemiological links and contacts to prevent further spread. Susceptible people, who have been exposed to measles, can avert disease if they receive measles vaccine within 72 hours of exposure or normal human immunoglobulin (NHIG) within seven days of exposure.

This was the first confirmed case of measles in NSW for over 5 months. As the diagnosis was confirmed more than 7 days after the child's flight, PHU staff contacted passengers seated in the same row and two rows fore and aft of the child to avoid infecting others should symptoms occur.

\section{Nine more cases}

Late in January 2008, Sydney West (SW) PHU reported three more cases of measles:

- Case 1, a 12-year-old boy, partially vaccinated, reported symptoms of fever, headache, cough and rash. Possible contacts were identified from a medical centre waiting room, cinema and the emergency department of hospital A.

- Case 2 was a 10-month-old unvaccinated boy. Symptoms included fever, conjunctivitis and coryza followed 2 days later by a rash. The infant had attended a religious function during his exposure period. Contacts included a GP waiting room where the case was isolated and the emergency department waiting room of hospital $\mathrm{A}$.

- Case 3, an 8-month-old unvaccinated girl, presented to the emergency department of hospital A with fever, cough, coryza, conjunctivitis and Koplik spots. A rash developed 2 days later. Possible contacts included attendees at a wedding and the emergency department waiting room of hospital A.

A clinic was held at hospital A for the susceptible contacts of these three cases who were offered measles-mumpsrubella (MMR) vaccine (22 contacts) and NHIG (four contacts).

SW PHU reported a further two cases in early February:

- Case 4, a woman in her 20s, presented to a GP with fever, sore throat and rash, and was later admitted to hospital B. Susceptible contacts were offered NHIG at a clinic held at hospital $\mathrm{B}$ and a medical centre in the area. Fifty-six susceptible persons received NHIG. 
- Case 5: on return from an overseas holiday, a woman in her 20s presented to the emergency department of hospital D with fever, cough, conjunctivitis, Koplik spots and rash. Contacts included her immediate family (two required NHIG) and work colleagues.

The following week, SW PHU was notified of a further three possible cases, only one of which, case 6 , was confirmed:

- Case 6, an unvaccinated 9-year-old girl presented to a GP with fever, cough, headache, coryza, photophobia and rash that had appeared three days later. Possible contacts of this girl were identified from primary school, after school care and school band. A clinic was held to offer NHIG prophylaxis: seven doses were provided including to the unvaccinated siblings of the girl with diagnosed measles.

Two children who attended the same child-care centre during their exposure period were notified in mid February:

- Case 7, an 11-month-old unvaccinated boy presented to a GP with cough, runny nose, conjunctivitis and fever followed by a rash three days later.

- Case 8, a 20-month-old unvaccinated boy presented to a GP with cough, coryza, fever and rash.

Another case was subsequently confirmed.

- Case 9 was a 13-year-old unvaccinated boy and the sibling of case 6 . This case had received NHIG prophylaxis on day 7 post-exposure and presented with modified clinical symptoms of headache, sore eyes and clammy skin followed after one day by a rash. A post-exposure clinic was held at the high school for susceptible contacts: 20 received NHIG.

All except one of the confirmed cases notified during this outbreak were unvaccinated. A third of those who were unvaccinated were too young for a first dose of MMR. The remaining cases either had no recollection of vaccination or had chosen not to be vaccinated.

\section{Further interventions}

In response to the outbreak, PHUs conducted five clinics for prophylaxis treatment for susceptible contacts throughout January and February. The majority of susceptible contacts received NHIG. GPs and emergency departments were alerted to the measles outbreak through the media and professional networks using a faxed measles alert. Media releases were used to alert the community about the risk of measles and to provide information about prevention, specifically the importance of immunisation. To date, no common exposures have been identified for this outbreak.

This outbreak highlights the impact of unvaccinated populations on the transmission of measles in the community. The modified presentation of case 9 is a reminder that atypical presentations and reduced prodromal periods may follow post-exposure prophylaxis.

\section{Meningococcal disease}

For January and February 2008, five cases of meningococcal disease were notified in NSW. All have survived. Of these, three were notified in January and two in February; three were males and three were under 5 years of age. One case was due to serogroup $\mathrm{C}$ (the vaccine-preventable strain) and three were due to serogroup B (for which there is no vaccine). For more information, see: http://www. health.nsw.gov.au/publichealth/infectious/index.asp.

\section{Enteric diseases}

In January 2008, NSW PHUs investigated 26 outbreaks of gastroenteritis, including 11 suspected foodborne outbreaks and 15 outbreaks suspected as been caused by person-to-person spread. The suspected foodborne outbreaks affected 61 people, ranging from 2 to 30 people per outbreak (median $=3$ ). No causal pathogens were identified. The NSW Food Authority inspected commercial premises associated with suspected outbreaks.

Nine of the suspected person-to-person outbreaks occurred in aged care facilities, affecting a total of 191 people, and six in child-care centres, affecting 44 people. Norovirus was confirmed in one aged-care facility outbreak, rotavirus was identified in one child-care facility outbreak; similar viruses were likely to have caused the other outbreaks.

In February, NSW PHUs investigated nine outbreaks of gastroenteritis, including one suspected foodborne outbreak and eight outbreaks suspected as been caused by person-to-person spread. The foodborne outbreak was a large outbreak likely caused by Bacillus cereus and Clostridium perfringens. Up to 75 people who attended a catered function were reportedly ill. Of the eight outbreaks suspected as been caused by person-to-person spread, five occurred in child-care settings, two in aged-care facilities and one in a hospital.

\section{Listeriosis}

Listeriosis is a rare but serious illness caused by eating food contaminated with bacteria called Listeria monocytogenes. Listeria bacteria are common in soil and some foods such as pre-cooked chicken, rare meats, cold delicatessen meats, raw seafood, smoked fish and soft cheeses. Eating foods that contain Listeria does not cause illness in most people. Some people, such as the elderly, pregnant women and people with weakened immune systems, are more susceptible to contracting the illness. Over the last 5 years, there have been between one and six notifications per month and between 22 and 28 cases a year.

In January 2008, nine cases of listeriosis were notified in NSW. Cases were aged from 0 to 90 years. Six died, 
including one stillborn baby. With the exception of the baby, those who died all had serious underlying illnesses. An epidemiological investigation found no links between the cases, and subsequent characterisation of the Listeria isolates from eight of the patients found distinct pulse field gel electrophoresis patterns. Although the number of cases is higher than expected for the month, available evidence indicates that the cases were not linked. No further cases have been reported through to mid February.

People who are pregnant or have immune dysfunction should avoid foods that may contain Listeria. For more information on listeriosis see: http://www.health.nsw.gov. $\mathrm{au} /$ factsheets/infectious/listeriosis.html and http://www. foodauthority.nsw.gov.au/consumer/pregnancy.asp.

\section{Increased arbovirus activity}

There have been increases in reported cases of Ross River virus and Barmah Forest virus infections in rural areas over summer. Both infections are spread by mosquitoes and may cause symptoms including fever, tiredness, rash and joint pain and swelling that last several days or longer. Murray Valley encephalitis (MVE) virus was identified in routine testing of mosquitoes trapped in early February near Griffith; however, chickens nearby have not tested positive for MVE and there is no evidence of human disease. Mosquitoes also transmit MVE, but human disease in NSW is rare. Most human infections do not lead to symptoms, but when they do occur they can be very serious. See: http://www.health.nsw.gov.au/factsheets/ infectious/murray_valley_enceph.html. The local PHU has initiated additional local surveillance via hospital emergency departments, local doctors and laboratories.
To prevent these infections, avoid being bitten by mosquitoes:

- Avoid being outside in the late afternoon and dusk.

- Wear loose fitting, light coloured clothing when outside that covers your arms and legs, and use an insect repellent that contains the chemical diethyl toluamide (DEET) or picardin.

- Fit fly screens to all windows, doors and chimneys, and keep them in good repair.

- Use a knockdown insecticide in bedrooms half an hour before going to bed. Use insecticides according to instructions.

- Ensure open containers of water are removed from around the house to prevent mosquitoes breeding.

- Cover the openings to water tanks with fine steel mesh to prevent mosquitoes from laying eggs in the tank.

- Take precautions when camping, such as using flyscreens on caravans and tents and by sleeping under mosquito-proof nets. Take particular care while fishing, ensuring that you follow personal precautions to avoid being bitten by mosquitoes.

\section{References}

1. NSW Health Department. NSW Health Department Notifiable Diseases Database System (NDD)(HOIST). North Sydney: Communicable Diseases Branch and Epidemiology and Surveillance Branch, NSW Health Department, 2008.

2. Heymann DL (editor). Control of communicable diseases manual. 18th edition. Washington DC: American Public Health Association, 2004. 
Figure 1. Reports of selected communicable diseases, NSW, January 2004 to February 2008, by month of onset.

Preliminary data: case counts in recent months may increase because of reporting delays.

Laboratory-confirmed cases only, except for measles, meningococcal disease and pertussis.

BFV, Barmah Forest virus infections; RRV, Ross River virus infections; Lab Conf, laboratory confirmed;

Men $\mathrm{Gp} C$ and $\mathrm{Gp} B$, meningococcal disease due to serogroup $C$ and serogroup B infection;

other/unk, other or unknown serogroups.

NB: multiple series in graphs are stacked, except gastroenteritis outbreaks.

NB: Outbreaks are more likely to be reported by nursing homes and hospitals than by other institutions.

\begin{tabular}{|lc|}
\hline \multicolumn{2}{|c|}{ NSW Population } \\
Male & $50 \%$ \\
$<5$ y & $7 \%$ \\
$5-24$ y & $27 \%$ \\
$25-64$ y & $53 \%$ \\
$65+$ y & $13 \%$ \\
Rural & $46 \%$ \\
\hline
\end{tabular}
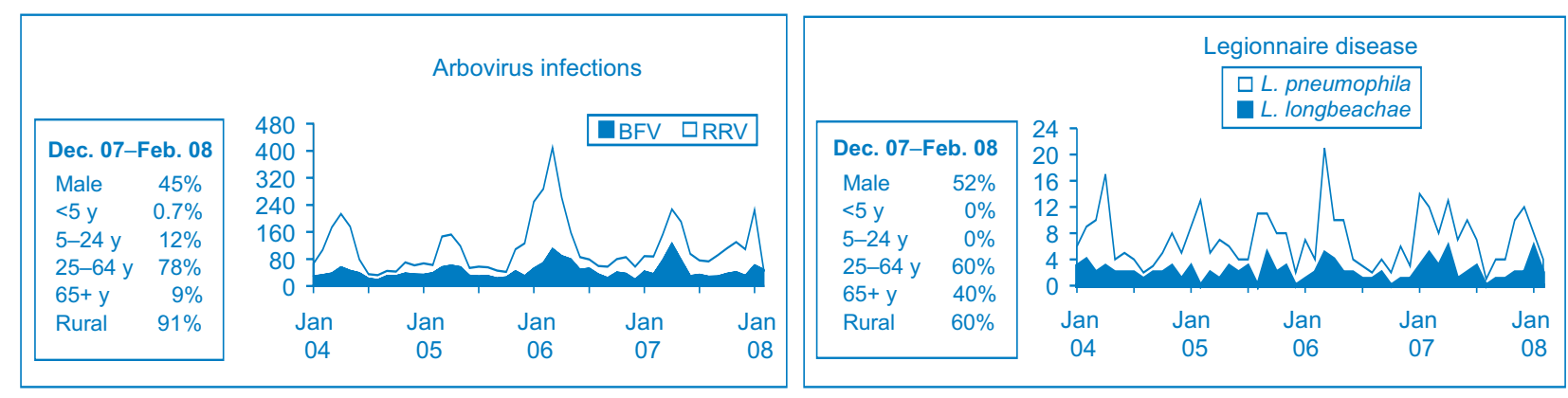

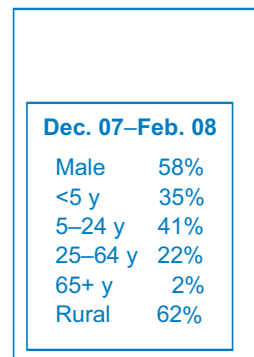

\section{Cryptosporidiosis}

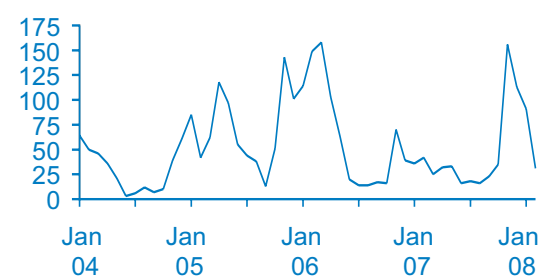

Gastroenteritis outbreaks in institutions
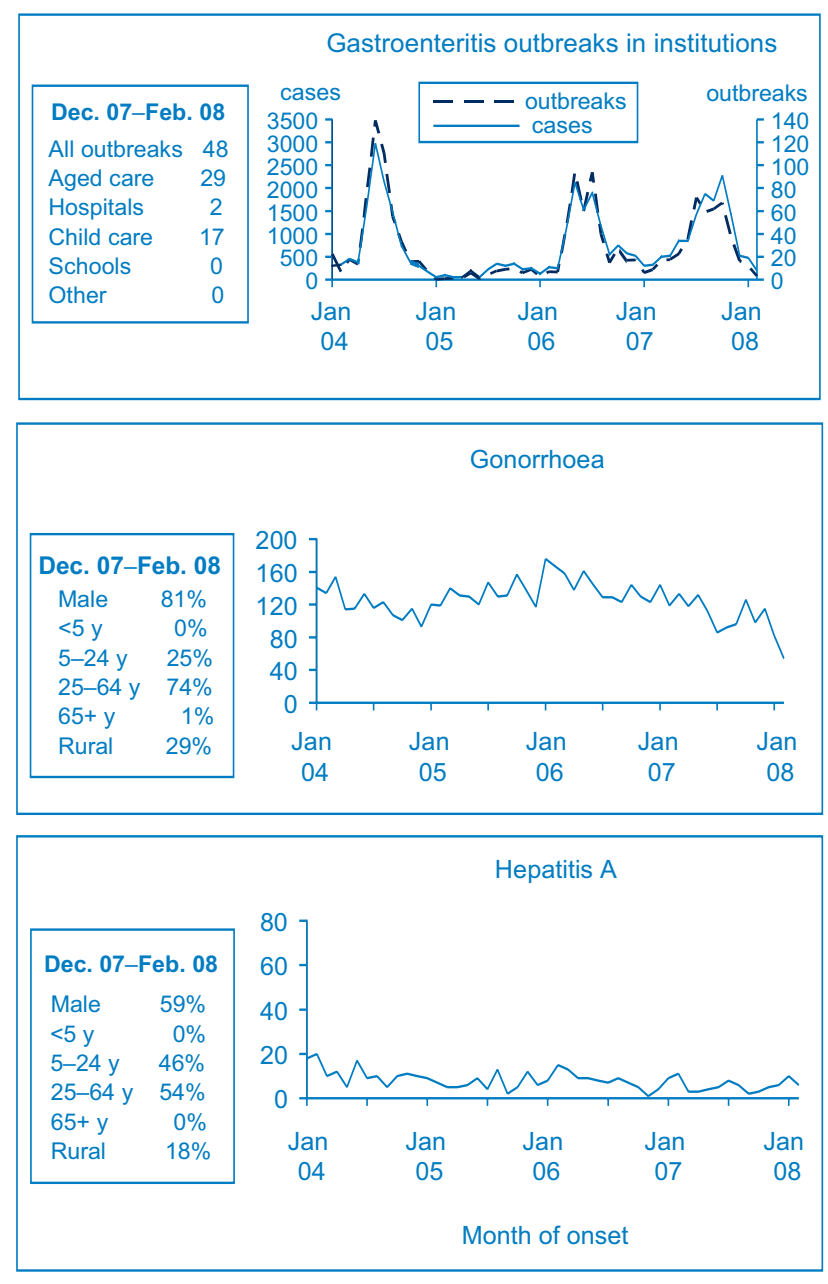
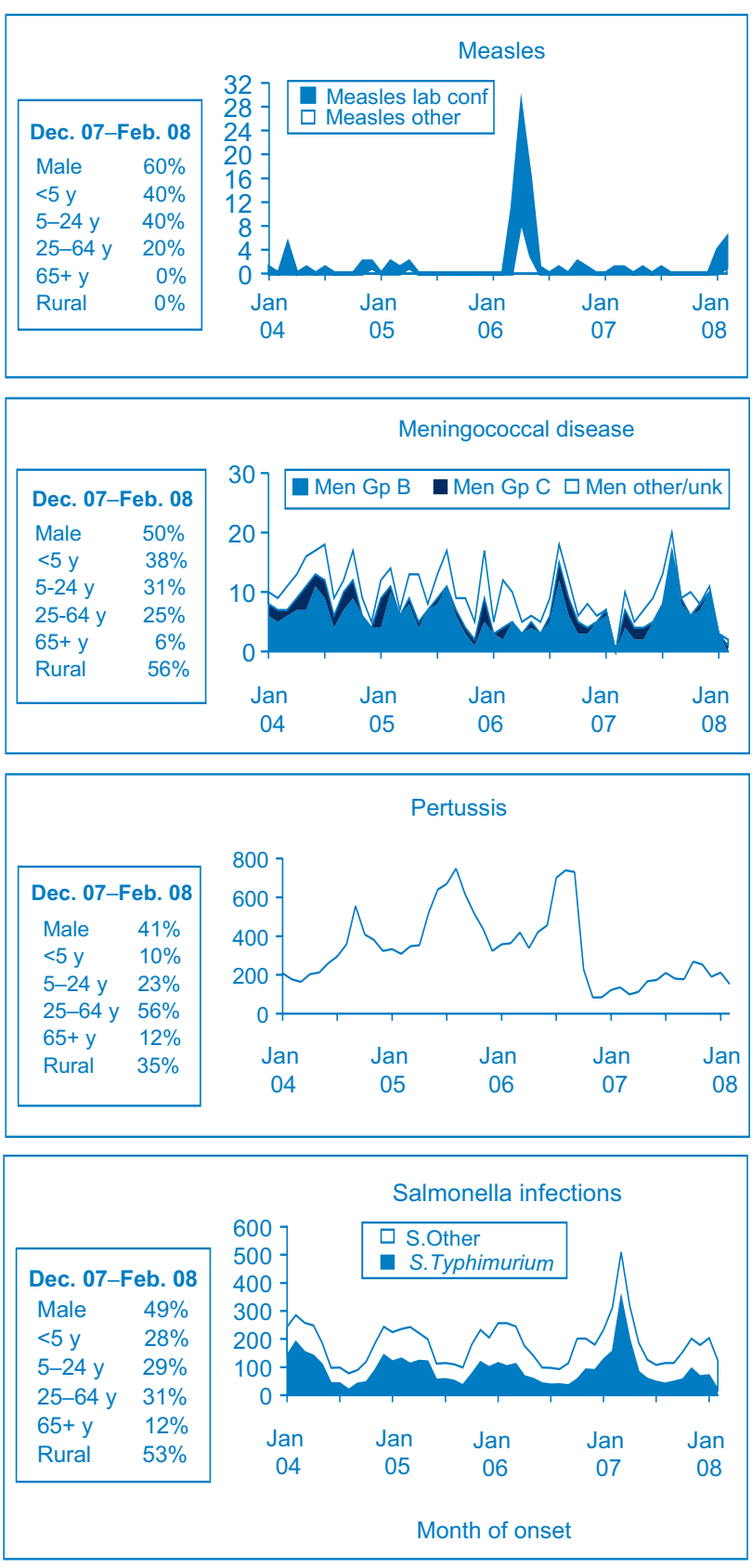
Table 1. Reports of notifiable conditions received in January 2008 by Area Health Services

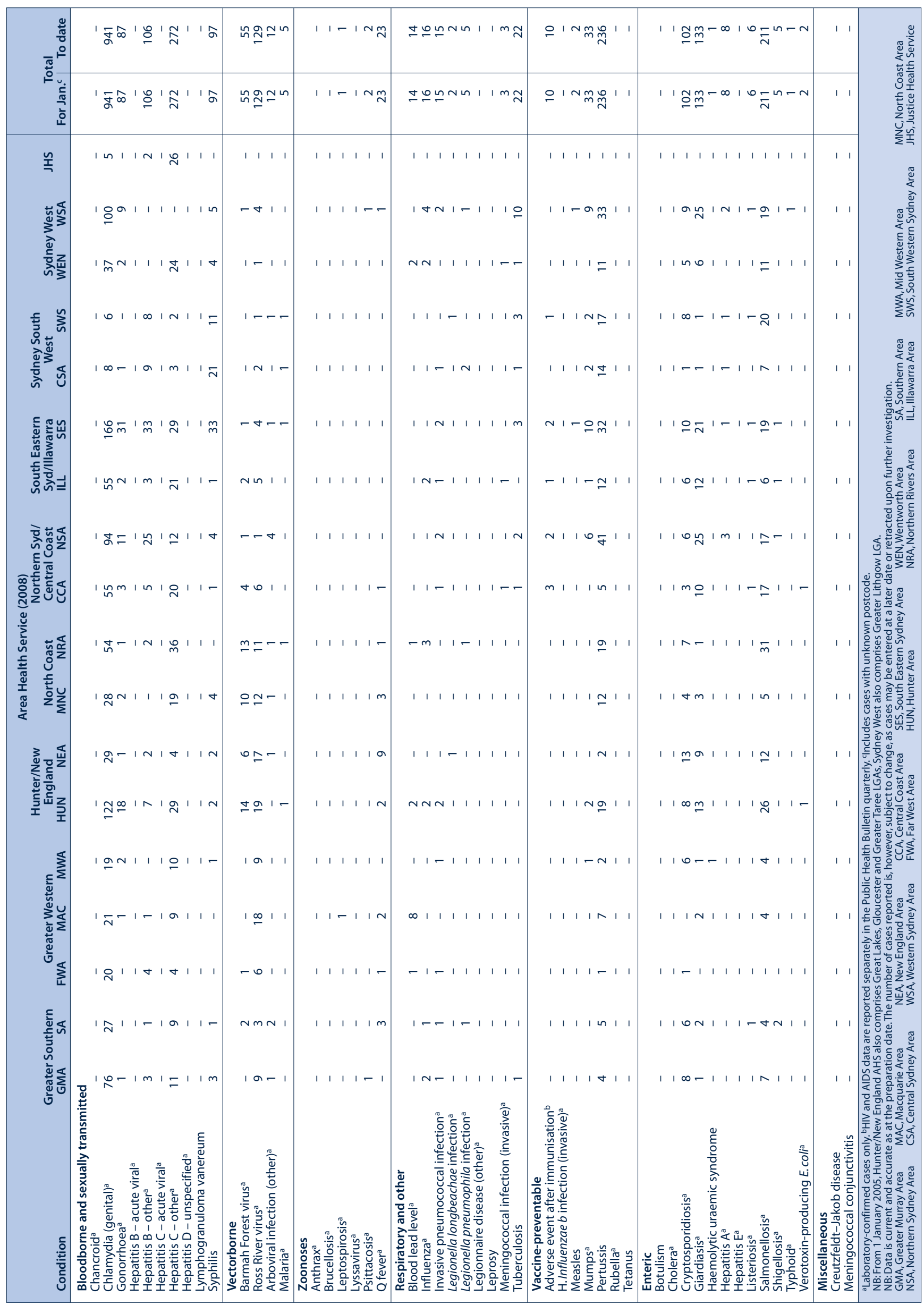


Table 2. Reports of notifiable conditions received in Febuary 2008 by Area Health Services

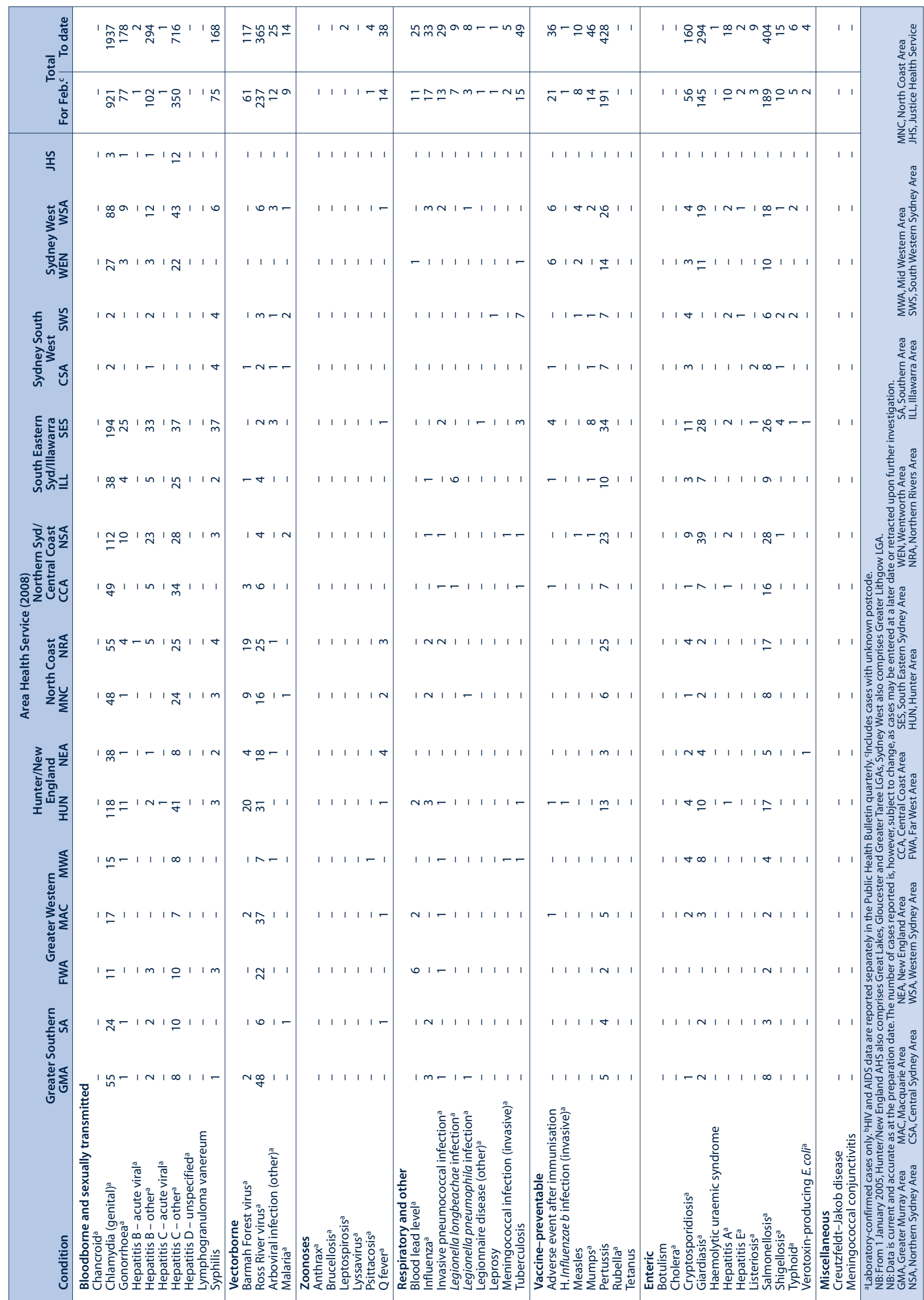

